\title{
Dephasing time and magnetoresistance of two-dimensional electron gas in spatially modulated magnetic fields
}

\author{
A. S. Mel'nikov, S. V. Mironov, and S. V. Sharov \\ Institute for Physics of Microstructures, Russian Academy of Science, 603950, Nizhniy Novgorod, GSP-105, Russia
}

(Dated: November 2, 2018)

\begin{abstract}
The effect of a spatially modulated magnetic field on the weak localization phenomenon in twodimensional electron gas (2DEG) is studied. Both the dephasing time $\tau_{H}$ and magnetoresistance are shown to reveal a nontrivial behavior as functions of the characteristics of magnetic field profiles. The magnetic field profiles with rather small spatial scales $d$ and modulation amplitudes $H_{0}$ such that $H_{0} d^{2} \ll \hbar c / e$ are characterized by the dephasing rate $\tau_{H}^{-1} \propto H_{0}^{2} d^{2}$. The increase in the flux value $H_{0} d^{2}$ results in a crossover to a standard linear dependence $\tau_{H}^{-1} \propto H_{0}$. Applying an external homogeneous magnetic field $H$ one can vary the local dephasing time in the system and affect the resulting average transport characteristics. We have investigated the dependence of the average resistance vs the field $H$ for some generic systems and predict a possibility to observe a positive magnetoresistance at not too large $H$ values. The resulting dependence of the resistance vs $H$ should reveal a peak at the field values $H \sim H_{0}$.
\end{abstract}

PACS numbers: 73.23.-b, 73.20.Fz, 73.50.-h, 73.43.Qt, 74.78.Na.

\section{INTRODUCTION}

The possibility to govern the electronic transport by applying an inhomogeneous magnetic field has recently attracted considerable interest. In particular, this problem is intensively studied in the hybrid ferromagnet/superconductor structures where the inhomogeneous magnetic field induced by the domain structure in the ferromagnet or a magnetic dot array is used to control the superconducting order parameter structure and the transport of Cooper pairs (see, e.g., Ref. 1, 2 for review). It is important to note that the typical values of the fields used in such experiments can be relatively small: $H \sim 10-10^{3} \mathrm{Oe}$. Nevertheless in the vicinity of the superconducting transition even these field values allow to destroy the Cooper pairs and, thus, strongly affect the electronic transport.

Another possibility to change the conductance applying relatively weak magnetic fields can be realized even in the normal (i.e., nonsuperconducting) structures provided we consider the systems with measurable quantum interference effects, e.g., disordered two-dimensional electron gas (2DEG) at low temperatures $T$. In the latter case the electron conductance is known to be affected by the weak-localization effects, which are caused by the quantum interference between the electronic waves propagating along different time-reversed quasiclassical trajectories ${ }^{3}$. The weak-localization correction $\Delta g$ to the Drude conductance $g_{D}$ in the diffusive limit can be written in the form

$$
\Delta g(\mathbf{r})=-\frac{2 e^{2}}{\pi \hbar} D \int_{\tau}^{\infty} W\left(\mathbf{r}, t_{0}\right) d t_{0}
$$

Here $W\left(\mathbf{r}, t_{0}\right) d t_{0}$ is the probability of electron return to the point $\mathbf{r}$ during the time interval $t_{0}<t<t_{0}+d t_{0}, \tau$ is the elastic scattering time, $D$ is the diffusion constant.
In the presence of an external magnetic field the probability of return is determined by the Green function $C\left(\mathbf{r}_{f}, t_{f}, \mathbf{r}_{i}, t_{i}\right)$ satisfying the so-called Cooperon equation:

$$
\begin{gathered}
W\left(\mathbf{r}, t_{0}\right)=C\left(\mathbf{r}, t_{i}+t_{0}, \mathbf{r}, t_{i}\right) \\
{\left[\frac{\partial}{\partial t_{f}}+D\left(-i \frac{\partial}{\partial \mathbf{r}_{f}}-\frac{2 e}{\hbar c} \mathbf{A}\left(\mathbf{r}_{f}\right)\right)^{2}+\frac{1}{\tau_{\varphi}}\right] C=} \\
=\delta\left(t_{f}-t_{i}\right) \delta\left(\mathbf{r}_{f}-\mathbf{r}_{i}\right),
\end{gathered}
$$

where $\mathbf{A}(\mathbf{r})$ is the vector potential, and $\tau_{\varphi}$ is the characteristic dephasing time. In the limit of zero magnetic field the expression for the weak-localization correction to the conductance obtained from the Eq. (3) takes the form:

$$
\Delta g(H=0)=-\frac{e^{2}}{2 \pi^{2} \hbar} \ln \frac{\tau_{\varphi}}{\tau} .
$$

The maximal size of closed trajectories contributing to this value is defined by the characteristic dephasing length $L_{\varphi}=\sqrt{D \tau_{\varphi}}$. Applying an external magnetic field perpendicular to the plane of 2DEG system one destroys the coherence for closed trajectories which enclose the magnetic flux larger than the flux quantum $\Phi_{0}=\pi \hbar c /|e|$. The resulting dephasing time $\tau_{H}$ becomes field dependent and can be obtained by comparing the flux through the contour of the size $\sqrt{D \tau_{H}}$ with $\Phi_{0}: \tau_{H}^{-1} \sim D H / \Phi_{0}$. As a consequence, the $2 \mathrm{DEG}$ system has a negative magnetoresistance (see Ref. $\underline{3}$ and references therein) and the conductance takes the form

$$
\begin{aligned}
& \Delta g=-\frac{e^{2}}{2 \pi^{2} \hbar} \\
& \times\left\{\psi\left(\frac{1}{2}+\frac{\hbar c}{4 e H D \tau}\right)-\psi\left(\frac{1}{2}+\frac{\hbar c}{4 e H D \tau_{\varphi}}\right)\right\},
\end{aligned}
$$


where $\psi$ is the digamma function. In the low field limit $\left(\tau_{H} \gg \tau_{\varphi}\right)$ the expression (5) transforms into the expansion

$$
\Delta g=-\frac{e^{2}}{2 \pi^{2} \hbar}\left\{\ln \frac{\tau_{\varphi}}{\tau}-\frac{2}{3}\left(\frac{e H D \tau_{\varphi}}{\hbar c}\right)^{2}+\ldots\right\} .
$$

Considering the magnetic fields which are modulated on microscopic length scales one should modify the above expressions taking into account the changes in the magnetic flux enclosed by the interfering trajectories passing through the regions with a rapidly changing magnetic field. The hybrid structures containing the 2DEG systems and certain sources of the spatially modulated magnetic fields attracted recently both the experimental and theoretical interest $4,5,6,7,8,9,10,11,12,13,14,15,16$. In part, these investigations have been stimulated by the possible potential of such systems for making detailed studies of the inhomogeneous magnetic field distributions. The magnetic field profiles with microscopic spatial scales can be induced, e.g., by a vortex lattice in a superconducting film $, \underline{4,6}, \underline{7}, \underline{.}$, as well as by a ferromagnetic film domain structure or a magnetic dot array positioned in the vicinity of the 2DEG system. Note also that the problem of the 2DEG conductance in a modulated magnetic field appears to be equivalent to the one of a rough 2DEG layer placed in a parallel magnetic field $\underline{10,11}$. For the particular case of vortices trapped in a superconducting film the magnetic field takes the form of flux tubes. An appropriate theoretical description of the weak-localization phenomenon for different flux tube radii as compared to the $L_{\varphi}$ length has been developed in Ref. 15. The corresponding contribution to the magnetoconductance at low average fields $H$ appeared to be proportional to the vortex concentration, i.e. to the $|H|$ value, in contrast to the $H^{2}$ behavior in a uniform magnetic field. The numerical analysis of the conductance corrections for the case of a lattice of magnetic flux tubes for arbitrary relations between the tube radius and $L_{\varphi}$ was performed in Ref. 8. Experimentally these predictions have been confirmed in Refs. 5, 6 for GaAs/AlGaAs heterostructures.

One can expect that the standard expressions (5) and (6) for local conductance should hold even for the spatially modulated magnetic fields provided the characteristic spatial scale $d$ of such modulation is much larger than the size of the closed trajectories contributing to the conductance corrections. For a rather strong value of the $\mathrm{z}$-component of the local field $B(\mathbf{r})$ the latter size can be defined as a minimum of two lengths: (i) the dephasing length in the absence of the field $L_{\varphi}=\sqrt{D \tau_{\varphi}}$ and (ii) the length $L_{B}=\sqrt{D \tau_{B}}=\sqrt{\hbar c / e B(\mathbf{r})}$ which formally coincides with the textbook definition of the magnetic length. Here we denote the magnetic field component along the direction perpendicular to the plane of a 2DEG system as $B(\mathbf{r})=H+\delta H(\mathbf{r})$, where $H$ is the average field value. Thus, considering rather strong fields and/or not very low temperatures one can use the above expressions for local conductance substituting the function $B(\mathbf{r})$ instead of the homogeneous field $H$. This adiabatic picture obviously breaks down when the closed interfering trajectories pass through the regions with rapidly changing magnetic field which happens either near the zeros of magnetic field or in the limit $d \lesssim \min \left[L_{\varphi}, L_{B}\right]$. The dephasing length and time in this case are no longer determined by the local field value and their dependence on the field modulation amplitude $H_{0}$ can become rather unusual. In particular, for the magnetic fields with zero spatial average the dephasing time is proportional to the square of the field amplitude $\left(\tau_{B}^{-1} \propto H_{0}^{2}\right)$ which is in sharp contrast to the linear in $H$ behavior of the dephasing rate for homogeneous fields. For some model one-dimensional field profiles such unusual field dependence of the dephasing rate has been previously predicted in Ref. 9. Experimentally this behavior $\tau_{B}^{-1} \propto H_{0}^{2}$ has been observed in Ref. 10 for random magnetic field profiles.

One of the goals of the present work is to suggest an analytical description of the weak localization phenomenon in inhomogeneous magnetic field for a wide class of the field profiles. In Section II we consider different regimes of the weak localization which are realized in different regions of magnetic field parameters. Also in this section we demonstrate that in strong magnetic fields and/or at not very low temperatures the local approximation is applicable for calculation of the quantum correction to the conductance. In Section III we consider the regimes corresponding to the weak amplitude of magnetic field. In particular, in subsection IIIA we present the calculations of a natural measurable quantity, i.e., the conductance averaged over the system area. As a next step, we proceed with the description of the dephasing rate behavior vs characteristics of the modulated magnetic field for a wide class of the periodic field profiles (see Section (IIIB). In Section IV we consider the case of strong magnetic fields and show that the dependence of the magnetoresistance vs the average field value appears to reveal an unusual peak structure. An obvious reason for the non-monotonous behavior of the resistance vs the average field is associated with partial field compensation effect which occurs in the regions where the $z$-components of the average and local fields have the opposite signs. Thus, applying the external magnetic field to the system placed in a modulated field with zero average one can stimulate the interference effects in some regions of the sample. Depending on the particular shape of the field profile this effect can result in the negative or positive magnetoresistance of the sample. In other words, the 2DEG samples coupled with the subsystems inducing the inhomogeneous magnetic field can reveal a so-called "anti-localization" (see, e.g., Refs. 17, 18) phenomenon when we apply an external magnetic field $H$. The results and suggestions for possible experiments are summarized in Section V.

Hereafter we focus on the case of classically weak magnetic fields $(e B(\mathbf{r}) \tau / m c \ll 1)$. Indeed the diffusive approximation for the electron motion is applicable when 
$B \ll \Phi_{0} / l^{2}$. At such fields the cyclotron frequency $\omega_{c}=e B(\mathbf{r}) / m c \ll\left(\hbar / \varepsilon_{F} \tau\right) 1 / \tau \ll 1 / \tau\left(\varepsilon_{F}\right.$ is the Fermi energy). Thus, these magnetic fields affect only the interference corrections to the transport characteristics, and we disregard the inhomogeneous field effect on the Drude-type contribution to the conductance which has been previously studied in Refs, 19,20,21,22 on the basis of the semiclassical approach.

Note that all results obtained in this paper are valid not only for ideal 2DEG with zero thickness but also for quasi-two-dimensional electron systems with finite thickness $a$, which has to satisfy the condition $a \ll L_{\varphi}$. In this case one can define the field range, in which longitudinal components do not affect the weak-localization correction to the conductance, while the transverse component does. Indeed, the effect of a weak longitudinal component of magnetic field $H_{\|}$can be described by the renormalization of the characteristic dephasing time: the value $\tau_{\varphi}^{-1}$ has to be replaced by $\tau_{\varphi}^{-1}+\tau_{H_{\|}}^{-1}$ (see, e.g., Ref. $\underline{3}$ ), where

$$
\frac{1}{\tau_{H_{\|}}}=\frac{1}{3}\left(\frac{e H_{\|} a}{\hbar c}\right)^{2} D
$$

Thus, the influence of the longitudinal component becomes noticeable only for $H_{\|} \sim H_{\|}^{*} \sim \Phi_{0} / a L_{\varphi}$. This value is much larger than the characteristic value $H_{\perp}^{*} \sim$ $\Phi_{0} / L_{\varphi}^{2}$ of transverse component, which can strongly affect the weak-localization correction. Hereafter we assume $H_{\|} \ll H_{\|}^{*}$ and neglect the effect of longitudinal magnetic field components.

\section{DIFFERENT REGIMES OF WEAK LOCALIZATION AND POSSIBLE APPROXIMATE APPROACHES}

In this section we outline the approximate approaches which are used for describing dephasing regimes in different regions of the system's parameters.

In the presence of an inhomogeneous magnetic field the weak-localization correction to the conductance of 2DEG is determined by the interplay of three lengthscales: (i) dephasing length $L_{\varphi}$, which at low temperatures grows as $T^{-1 / 2}$ (see Ref. 24), (ii) the scale of the magnetic field inhomogeneity $d$, and (iii) the magnetic length $L_{H_{0}}=\sqrt{\Phi_{0} / H_{0}}$, where $H_{0}$ is the amplitude of the periodic magnetic field. The ratios of these lengths define the behaviour of the weak-localization correction to the conductance. For the analysis of the behavior of quantum correction to the conductance it is convenient to use the diagram shown in Fig. 1. We choose the parameter $d / L_{\varphi}$ to describe the temperature dependence of weak-localization correction to the conductance and the parameter $d^{2} / L_{H_{0}}^{2}$ to consider the influence of modulated magnetic field. Note that for a two-dimensional lattice with translational vectors $\mathbf{R}_{n}=n_{1} d_{1} \mathbf{a}_{1}+n_{2} d_{2} \mathbf{a}_{2}$ ( $\mathbf{a}_{1}, \mathbf{a}_{2}$ are unit vectors and $n_{1}, n_{2}$ are integers) the

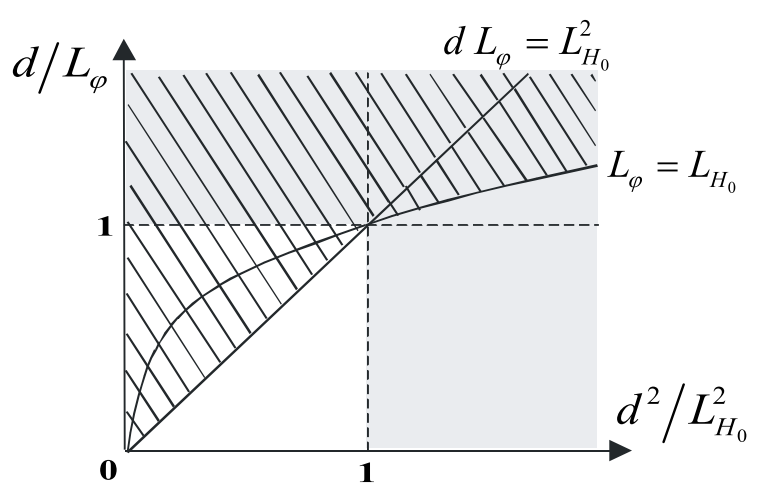

FIG. 1: The diagram of different weak-localization regimes in the plane of key parameters. In the gray region the scale of the magnetic field inhomogeneity $d$ is large enough so that the dephasing is controlled by the local magnetic field. In the white square the magnetic field is weak but its inhomogeneity reveals in the renormalization of the effective electron dephasing time. In the shaded region the dephasing occurs at the dephasing length $L_{\varphi}$ and the influence of magnetic filed reveals in a small additional correction to the conductivity.

value $d$ is the absolute value of the smallest vector $\mathbf{R}_{n}$ $\left(d=\min \left[d_{1}, d_{2}\right]\right)$.

Depending on the ratio $d / L_{H_{0}}$ there exist two qualitatively different mechanisms of the electron dephasing caused by the inhomogeneous field. Provided $d / L_{H_{0}}<1$ and simultaneously the $d$ length scale is smaller than $L_{\varphi}$ (white square in Fig. 1) the dephasing scenario in a modulated field with zero average can be explained by the following qualitative arguments. Let us consider a certain quasiclassical trajectory of the length $L=\sqrt{D \tau_{B}}$ which encloses many primitive cells of the periodic field profile. The magnetic flux coming from the cells which are positioned inside the contour appears to be averaged to zero. The only residual flux is associated with the cells which are crossed by the quasiclassical trajectory and give a flux contribution which strongly fluctuates with the increase in the area enclosed by the trajectory. The characteristic amplitude of these flux fluctuations can be estimated as the number of the elementary cells crossed by the trajectory $(L / d)$ multiplied by the typical flux value $H_{0} d^{2}: \delta \Phi \sim L d H_{0}$. Comparing this fluctuating flux with the flux quantum $\Phi_{0}$ we find the length of the dephasing $L \sim \Phi_{0} / d H_{0} \sim L_{H_{0}}^{2} / d$ and corresponding dephasing rate $\tau_{B}^{-1} \sim D d^{2} H_{0}^{2} / \Phi_{0}^{2}$. These qualitative arguments are in beautiful agreement with the quantitative consideration in section $1 \mathrm{IIB}$ carried out on the basis of the "nearly free electron" approximation. In the opposite limit $d / L_{H_{0}}>1$ the dephasing is controlled by the local magnetic field value.

Of course, the magnetic field provides a dominating dephasing mechanism only at low temperatures. For rather high temperatures when $L_{H_{0}}>\max \left[L_{\varphi}, \sqrt{d L_{\varphi}}\right]$ (shaded region in Fig. 1) the dephasing occurs at the 
length $L_{\varphi}$ and one can analyze the magnetic field effect on the weak-localization correction to the conductance perturbatively (see section IIIA).

The gray region in Fig. $1\left(d \gg L_{H_{0}}\right.$ or $\left.d>L_{\varphi}\right)$ corresponds to another important regime: the weaklocalization correction to the conductance in this case can be obtained within the local approximation. This means that the conductivity at each point of the sample depends on the local magnetic field. The validity of the local approximation in this regime can be shown directly from the Eq. (3). Let us introduce the vectors

$$
\mathbf{R}=\frac{\mathbf{r}_{f}+\mathbf{r}_{i}}{2}, \quad \mathbf{r}=\mathbf{r}_{f}-\mathbf{r}_{i}
$$

An electron is dephased at the lengthscale which is the minimum of the scales $L_{\varphi}$ and $L_{H_{0}}$, i.e. only the region $|\mathbf{r}|<\min \left[L_{\varphi}, L_{H_{0}}\right]$ makes the contribution to the weaklocalization correction to the conductance. Therefore in the limit $d \gg \min \left[L_{\varphi}, L_{H_{0}}\right]$ it is necessary to find the solution of Eq. (3) only in the case when $|\mathbf{r}| \ll d$. In this case we can expand the vector potential $\mathbf{A}\left(\mathbf{r}_{f}\right)$ :

$$
\mathbf{A}\left(\mathbf{r}_{f}\right)=\mathbf{A}\left(\mathbf{R}+\frac{\mathbf{r}}{2}\right) \approx \mathbf{A}(\mathbf{R})+\frac{1}{2}\left(\mathbf{r}, \frac{\partial}{\partial \mathbf{R}}\right) \mathbf{A}(\mathbf{R}) .
$$

Then after introducing a modified Green function

$$
\tilde{C}(\mathbf{R}, \mathbf{r})=C(\mathbf{R}, \mathbf{r}) \exp \left[-\frac{2 i e}{\hbar c}(\mathbf{A}(\mathbf{R}), \mathbf{r})\right]
$$

one can obtain the following equation :

$$
\begin{array}{r}
{\left[\frac{\partial}{\partial t_{f}}+D\left(-i \frac{\partial}{\partial \mathbf{r}}-\frac{i}{2} \frac{\partial}{\partial \mathbf{R}}-\frac{2 e}{\hbar c} \tilde{\mathbf{A}}(\mathbf{R}, \mathbf{r})\right)^{2}+\frac{1}{\tau_{\varphi}}\right] \tilde{C}=} \\
=\exp \left[-\frac{2 i e}{\hbar c}(\mathbf{A}(\mathbf{R}), \mathbf{r})\right] \delta(\mathbf{r}) \delta\left(t_{f}-t_{i}\right)
\end{array}
$$

where

$$
\tilde{\mathbf{A}}(\mathbf{R}, \mathbf{r})=\frac{1}{2}[\tilde{\mathbf{H}}(\mathbf{R}), \mathbf{r}], \quad \tilde{\mathbf{H}}(\mathbf{R})=\left[\frac{\partial}{\partial \mathbf{R}}, \mathbf{A}(\mathbf{R})\right] .
$$

The right part of Eq. (17) contains $\delta(\mathbf{r})$, so we can put $\mathbf{r}=0$ in the exponential prefactor.

Note, that in Eq. (17) one can neglect the term containing the derivative $\partial / \partial \mathbf{R}$. Indeed, it has the order $d^{-1}$ whereas the value $\tilde{\mathbf{A}}(\mathbf{R}, \mathbf{r})$ and the term containing the derivative $\partial / \partial \mathbf{r}$ have the order $1 / \min \left[L_{\varphi}, L_{H_{0}}\right]$, so the terms containing $\partial / \partial \mathbf{R}$ are negligible. In this case the Eq. (17) takes the form

$$
\begin{array}{r}
{\left[\frac{\partial}{\partial t_{f}}+D\left(-i \frac{\partial}{\partial \mathbf{r}}-\frac{2 e}{\hbar c} \tilde{\mathbf{A}}(\mathbf{R}, \mathbf{r})\right)^{2}+\frac{1}{\tau_{\varphi}}\right] \tilde{C}=} \\
=\delta\left(t_{f}-t_{i}\right) \delta(\mathbf{r})
\end{array}
$$

The equation (8) formally coincides with the Eq. (3) for the case of homogeneous magnetic field $\tilde{\mathbf{H}}(\mathbf{R})$, which depends on the variable $\mathbf{R}$ as a parameter. Thus, in the gray region in the Fig. 1 one can use the local approximation to calculate the weak-localization correction to the conductance. In what follows we show that in this case the spatially modulated magnetic field with zero average can cause the effect of positive magnetoresistance of $2 \mathrm{DEG}$ in the external homogeneous magnetic field.

Note, that the local approximation breaks down at the points where the magnetic field is changing rapidly, i.e. near the zeros of the magnetic field. Nevertheless considering the spatially averaged conductance one can neglect the correction coming from these regions which appears to be small in the limit $L_{\varphi} / d \ll 1$.

\section{WEAK MAGNETIC FIELDS. NEGATIVE MAGNETORESISTANCE}

\section{A. Magnetoresistance of 2DEG. Second order perturbation theory.}

1. Quantum correction to the conductance in the field with arbitrary spatial configuration

Let us consider the case of magnetic field with arbitrary spatial configuration but with zero spatial average. In this subsection we find an analytical solution of the equation (3) in the extreme case of low magnetic field. This means that the magnetic flux through any closed contour of the size $\sqrt{D \tau_{\varphi}}$ is mush less than $\Phi_{0}$. In this case the magnetic field weakly affects the weak-localization correction to the conductance, and the equation (3) can be solved within the frames of the perturbation theory with a small parameter proportional to the value of magnetic field.

Let us introduce the Fourier transform of the magnetic field:

$$
H_{z}(\mathbf{r})=\int_{-\infty}^{\infty} \int_{-\infty}^{\infty} H_{\mathbf{k}} e^{i \mathbf{k r}} d^{2} \mathbf{k}
$$

Here $\mathbf{r}$ is a vector in the plane of 2DEG. We assume all spatial harmonics $H_{\mathbf{k}}$ of magnetic field to be small.

The corresponding vector potential can be chosen in the form

$$
\mathbf{A}(\mathbf{r})=\int_{-\infty}^{\infty} \int_{-\infty}^{\infty} \mathbf{A}_{\mathbf{k}} e^{i \mathbf{k r}} d^{2} \mathbf{k}
$$

where

$$
\mathbf{A}_{\mathbf{k}}=\frac{i\left[\mathbf{k}, \mathbf{z}_{0}\right]}{k^{2}} H_{\mathbf{k}}
$$

In the zero order of the small parameter we considered the Green function as the one without magnetic field: 


$$
C_{0}=\frac{1}{4 \pi D t_{0}} \exp \left(-\frac{r_{0}^{2}}{4 D t_{0}}-\frac{t_{0}}{\tau_{\varphi}}\right) \text {. }
$$

Here $\mathbf{r}_{0}=\mathbf{r}_{f}-\mathbf{r}_{i}, t_{0}=t_{f}-t_{i}$. Further we represent the operator in the left part of the Eq. (3) as a sum of operators $\hat{F}=\hat{F}_{0}+\hat{F}_{1}+\hat{F}_{2}$ where

$$
\begin{gathered}
\hat{F}_{0}=\left[\frac{\partial}{\partial t_{f}}-D \frac{\partial^{2}}{\partial \mathbf{r}_{f}^{2}}+\frac{1}{\tau_{\varphi}}\right] \\
\hat{F}_{1}=\frac{4 e D}{\hbar c} \int_{-\infty}^{\infty} \int_{-\infty}^{\infty} d^{2} \mathbf{k} H_{\mathbf{k}} e^{i \mathbf{k r}_{f}}\left[\mathbf{k}, \frac{\partial}{\partial \mathbf{r}_{f}}\right], \\
\hat{F}_{2}=\frac{4 e^{2} D}{\hbar^{2} c^{2}}\left[\int_{-\infty}^{\infty} \int_{-\infty}^{\infty} d^{2} \mathbf{k} \frac{i\left[\mathbf{k}, \mathbf{z}_{0}\right]}{k^{2}} H_{\mathbf{k}} e^{i \mathbf{k r}}\right]^{2} .
\end{gathered}
$$

In the first order of perturbation theory the correction to the Green function can be written as

$$
\begin{gathered}
C_{1}\left(\mathbf{r}_{f}, t_{f}, \mathbf{r}_{i}, t_{i},\right)= \\
=-\int_{t_{i}}^{t_{f}} \int_{-\infty}^{+\infty} \int_{-\infty}^{+\infty} C_{0}\left(\mathbf{r}_{f}, t_{f}, \mathbf{r}^{\prime}, t^{\prime}\right) \hat{F}_{1} C_{0}\left(\mathbf{r}^{\prime}, t^{\prime}, \mathbf{r}_{i}, t_{i}\right) d \mathbf{r}^{\prime} d t^{\prime}
\end{gathered}
$$

If $\left(x_{f}, y_{f}\right) \rightarrow\left(x_{i}, y_{i}\right)$ then $C_{1}=0$. This reflects the fact that the quantum correction does not depend on the sign of applied magnetic field. The second order correction to the Green function is defined by the expression

$$
\begin{gathered}
C_{2}=-\int_{t_{i}}^{t_{f}} \int_{-\infty}^{+\infty} \int_{-\infty}^{+\infty} C_{0}\left(\mathbf{r}_{f}, t_{f}, \mathbf{r}^{\prime}, t^{\prime}\right) \\
\times\left[\hat{F}_{1} C_{1}\left(\mathbf{r}^{\prime}, t^{\prime}, \mathbf{r}_{i}, t_{i}\right)+\hat{F}_{2} C_{0}\left(\mathbf{r}^{\prime}, t^{\prime}, \mathbf{r}_{i}, t_{i}\right)\right] d \mathbf{r}^{\prime} d t^{\prime} .
\end{gathered}
$$

Let us introduce the value $\Delta g_{H}$

$$
\Delta g_{H}=\Delta g(\mathbf{B})-\Delta g(0)
$$

where the $\Delta g(\mathbf{B})$ is the weak-localization correction to the conductance in the inhomogeneous magnetic field $\mathbf{B}(\mathbf{r})$. Then the value $\Delta g_{H}$ is determined by the second order correction $C_{2}\left(\mathbf{r}, t_{0}\right)$ :

$$
\Delta g_{H}(\mathbf{r})=-\frac{2 e^{2} D}{\pi \hbar} \int_{0}^{\infty} C_{2}\left(\mathbf{r}, t_{0}\right) d t_{0} .
$$

In the expression (14) we put the lower integration limit equal to zero because of the absence of the small $t_{0}$ divergence in the integrand. Thus, we neglect the correction of the order $\tau / \tau_{\varphi}$.

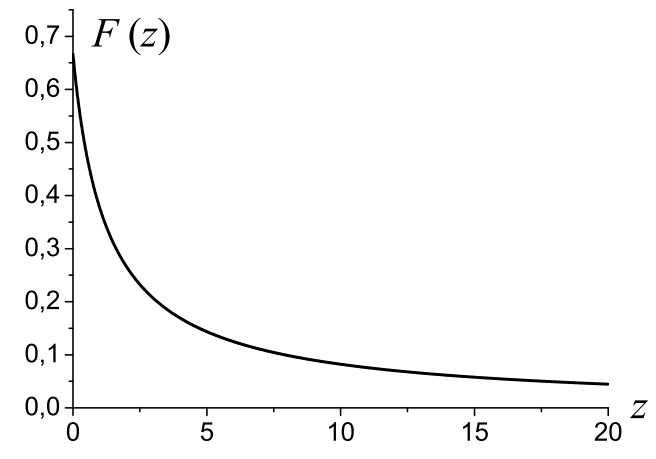

FIG. 2: The function $F(z)$ defined by the expression (18).

As we are interested only in spatially averaged correction to the conductance the expression (14) should be integrated over $\mathbf{r}$. Performing the integration in the expression (12) we obtain the averaged correction to the conductance $\left\langle\Delta g_{H}\right\rangle$ :

$$
\begin{gathered}
\left\langle\Delta g_{H}\right\rangle=\frac{8 e^{4} D}{\hbar^{3} c^{2} S} \int_{0}^{\infty} d t_{0} e^{-\frac{t_{0}}{\tau_{\varphi}}} \int_{-\infty}^{\infty} \int_{-\infty}^{\infty} d^{2} \mathbf{k} \frac{H_{\mathbf{k}} H_{-\mathbf{k}}}{k^{2}} \\
\times\left(1-\frac{2}{k \sqrt{D t_{0}}} e^{-\frac{k^{2} D t_{0}}{4}} \Phi\left(\frac{k \sqrt{D t_{0}}}{2}\right)\right)
\end{gathered}
$$

where $S$ is the area of the sample, $\Phi(\xi)=\int_{0}^{\xi} e^{t^{2}} d t$. Since we neglect the corrections proportional to $\tau / \tau_{\varphi}$ the integration in the expression (15) should be performed over $|\mathbf{k}|<(D \tau)^{-1 / 2}$.

Note that $H_{-\mathbf{k}}=H_{\mathbf{k}}^{*}$ and for $\alpha>1$

$$
\int_{0}^{\infty} e^{-\alpha x^{2}} \Phi(x) d x=\frac{1}{4 \sqrt{\alpha}} \ln \left(\frac{\sqrt{\alpha}+1}{\sqrt{\alpha}-1}\right) .
$$

Then the expression (15) can be rewritten in the form

$$
\left\langle\Delta g_{H}\right\rangle=\frac{2 e^{4} D^{2} \tau_{\varphi}^{2}}{\hbar^{3} c^{2} S} \int_{-\infty}^{\infty} \int_{-\infty}^{\infty} d^{2} \mathbf{k}\left|H_{\mathbf{k}}\right|^{2} F\left(\frac{k^{2} D \tau_{\varphi}}{4}\right)
$$

where

$$
F(z)=\frac{1}{z}\left(1-\frac{1}{\sqrt{z(z+1)}} \ln (\sqrt{z+1}+\sqrt{z})\right) .
$$

With the increase in the $z$ coordinate the function $F(z)$ is monotonically decreasing from the value $2 / 3$ at $z=0$ to zero at $z=\infty$ decaying as $z^{-1}$ at large $z$ values. Therefore, the spatial harmonics of magnetic field with $|\mathbf{k}| \ll L_{\varphi}^{-1}$ make the main contribution to the weaklocalization correction. In particular, for the case of 
magnetic field with narrow spectrum in the momentum space (the value $H_{\mathbf{k}}$ is non-zero only in the spectral region $\left.|\mathbf{k}| \ll L_{\varphi}^{-1}\right)$ the value $\left\langle\Delta g_{H}\right\rangle$ is defined by the expression

$$
\left\langle\Delta g_{H}\right\rangle=\frac{4 e^{4} D^{2} \tau_{\varphi}^{2}}{3 \hbar^{3} c^{2} S} \int_{-\infty}^{\infty} \int_{-\infty}^{\infty}\left|H_{\mathbf{k}}\right|^{2} d^{2} \mathbf{k} .
$$

Using the properties of Fourier transformation, we can rewrite the expression (19) in the form

$$
\left\langle\Delta g_{H}\right\rangle=\frac{e^{4} D^{2} \tau_{\varphi}^{2}}{3 \pi^{2} \hbar^{3} c^{2} S} \int_{S_{0}} H_{z}^{2} d^{2} \mathbf{r} .
$$

It is seen from the Eq. (20) that in the case of weak nonhomogeneous field with spatial scale larger than $L_{\varphi}$ the averaged correction to conductance is defined only by the square of magnetic field averaged over the sample. This result corresponds to the local approximation.

Note that the expression (15) is correct also in the case of magnetic field with non-zero spatial average $H$ which satisfies the condition $H L_{\varphi}^{2} \ll \Phi_{0}$. In this case the expression for the spatially averaged weak-localization correction to the conductance has the form

$$
\begin{aligned}
\langle g(H)\rangle & =g_{D}-\frac{e^{2}}{2 \pi^{2} \hbar} \ln \frac{\tau_{\varphi}}{\tau}+\left\langle\Delta g_{H}\right\rangle \\
& +\frac{e^{2}}{3 \pi^{2} \hbar}\left(\frac{e H D \tau_{\varphi}}{\hbar c}\right)^{2},
\end{aligned}
$$

where $\left\langle\Delta g_{H}\right\rangle$ is defined by the expression (17) for magnetic field with zero average. Thus the homogeneous component of the magnetic field makes small additional contribution to the averaged correction.

The expression (14) for the local conductance value can be further simplified for the particular case of onedimensional field which depends on the $x$ coordinate. In this case the magnetic field can be written in the form

$$
H_{z}=\int_{-\infty}^{\infty} H_{k} e^{i k x} d k
$$

where $k$ is the scalar Fourier variable. Performing integration in (12) we obtain an analytical expression for the Green function $C_{2}(\mathbf{r})$ :

$$
\begin{aligned}
& C_{2}=\frac{4 e^{2} e^{-\frac{t_{0}}{\tau_{\varphi}}}}{\pi \hbar^{2} c^{2}\left(D t_{0}\right)^{3 / 2}} \int_{-\infty}^{\infty} d k \int_{-\infty}^{\infty} d q \frac{H_{k} H_{q}}{k^{2} q^{2}(k+q)} e^{i(k+q) x} \\
& \times\left[e^{-\frac{(q+k)^{2} D t_{0}}{4}} \Phi\left(\frac{(k+q) \sqrt{D t_{0}}}{2}\right)\right. \\
& -e^{-\frac{D k^{2} t_{0}}{4}} \Phi\left(\frac{k \sqrt{D t_{0}}}{2}\right)-e^{-\frac{q^{2} D t_{0}}{4}} \Phi\left(\frac{q \sqrt{D t_{0}}}{2}\right) \\
& \left.+\frac{k q D t_{0}}{2} e^{-\frac{(k+q)^{2} D t_{0}}{4}} \Phi\left(\frac{(k+q) \sqrt{D t_{0}}}{2}\right)\right] .
\end{aligned}
$$

This expression can be made even more transparent in the special case of the magnetic field with the sinusoidal profile.

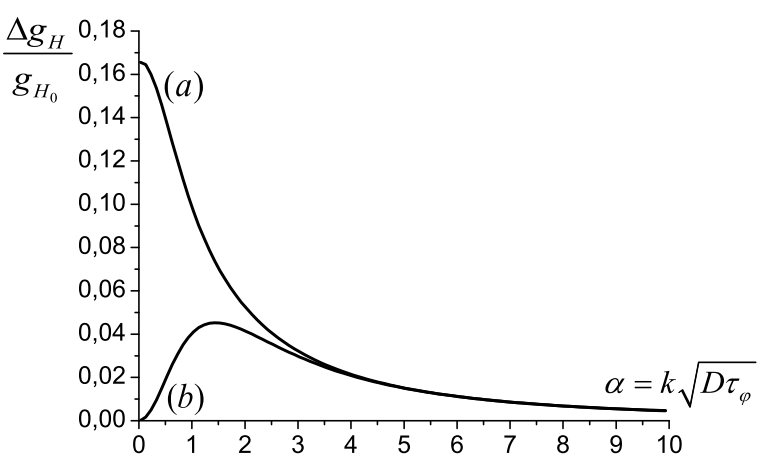

FIG. 3: The dependence of the value $\Delta g_{H}$ vs parameter $\alpha$ for $x=d / 2$ (a) and $x=d / 2(\mathrm{~b})$.

2. Quantum correction to the conductance in low sinusoidal magnetic field

Let the magnetic field has the form

$$
H_{z}(x)=H_{0} \cos (k x) .
$$

Then for the value $\Delta g_{H}$ we obtain the following expression:

$$
\begin{gathered}
\Delta g_{H}=\frac{2 e^{4} H_{0}^{2}}{\pi^{2} \hbar^{3} c^{2} k^{4}} \int_{0}^{\infty} e^{-\frac{\beta^{2}}{k^{2} D \tau_{\varphi}}}\left(\beta-2 e^{-\frac{\beta^{2}}{4}} \Phi\left(\frac{\beta}{2}\right)\right. \\
\left.+\cos (2 k x) \frac{e^{-\beta^{2}}}{\beta^{2}}\left\{4 e^{\frac{3}{4} \beta^{2}} \Phi\left(\frac{\beta}{2}\right)-\left(\beta^{2}+2\right) \Phi(\beta)\right\}\right) d \beta .
\end{gathered}
$$

One can see that the only dimensionless parameter $\alpha=$ $k \sqrt{D \tau_{\varphi}}=\pi L_{\varphi} / d$ defines the value of the integral in the expression (24).

It is interesting to consider the dependence of the value $\Delta g_{H}$ on the period of magnetic field $d$. The dependencies of the value $\Delta g_{H}$ on the parameter $\alpha=\pi L_{\varphi} / d$ for two interesting cases $x=0$ and $x=d / 2$ are shown in Fig. 3, where we introduced the value

$$
g_{H_{0}}=\frac{2 e^{2}}{\hbar}\left(\frac{H_{0} D \tau_{\varphi}}{\Phi_{0}}\right)^{2}
$$

Note that the value $\Delta g_{H}(\mathbf{r})$ is defined by the module of the averaged magnetic flux through all possible closed trajectories of the size $L_{\varphi}$ which are passing through the point $\mathbf{r}$. In the maxima of the magnetic field $(x=n d$, $n$ is an integer) the averaged flux is decreasing with $d$ decreasing (increasing $\alpha$ ) and this leads to the decrease in the $\Delta g_{H}$ value. At the zeroes of the magnetic field $(x=$ $d / 2+n d)$ when the scales $d$ and $L_{\varphi}$ are comparable one can observe a maximum in the dependence $\Delta g_{H}$ vs $\alpha$ (see 
the curve (b) in Fig. (3). In this case the averaged module of the magnetic flux through the closed trajectories is maximal.

The analysis of the expression for $\Delta g_{H}$ shows that in the limit of $d \ll L_{\varphi}$ the value $\Delta g_{H}$ is proportional to $d^{2}$ :

$$
\Delta g_{H} \approx \frac{e^{2}}{\pi^{2} \hbar}\left(\frac{L_{\varphi}}{L_{H_{0}}}\right)^{4} \cdot\left(\frac{d}{L_{\varphi}}\right)^{2}
$$

where $L_{H_{0}}=\sqrt{\Phi_{0} / H_{0}}$.

In case of smooth field variation $\left(d \gg L_{\varphi}\right.$, but $\left.H_{0} d L_{\varphi} \ll \Phi_{0}\right)$ keeping the corrections $\propto \alpha^{2}$ we find:

$$
\Delta g_{H}=\frac{e^{2} L_{\varphi}^{4}}{6 \hbar L_{H_{0}}^{4}}\left((1+\cos 2 k x)-\frac{\alpha^{2}}{5}(1+7 \cos 2 k x)\right) .
$$

The expression (25) differs strongly from the correspondent expression for the case of homogeneous field, since even at the points of zero magnetic field the value $\Delta g_{H}$ is positive. This fact is quite natural since the averaged module of the flux through the closed trajectories does not vanish even at these points.

\section{B. Dephasing time in spatially periodic magnetic fields}

The expressions (17) and (21) obtained within the perturbation theory diverge at low temperatures as the dephasing time $\tau_{\varphi}$ tends to infinity. Thus, to describe the behavior of the conductance at low temperatures one should take account of the renormalization of the dephasing time caused by the magnetic field.

In this subsection we consider such renormalization procedure for a periodic magnetic field

$$
H_{z}\left(\mathbf{r}+\mathbf{R}_{n}\right)=H_{z}(\mathbf{r}),
$$

where $\mathbf{R}_{n}$ are translational vectors which generate a twodimensional lattice.

The expression for the electron probability of return obtained from the solution of the equation (3) can be written in the following form:

$$
W\left(\mathbf{r}, t_{0}\right)=e^{-\frac{t_{0}}{\tau_{\varphi}}} \sum_{j}\left|\psi_{j}(\mathbf{r})\right|^{2} e^{-\varepsilon_{j} D t_{0}} .
$$

Here $\varepsilon_{j}$ and $\psi_{j}(\mathbf{r})$ are the eigenvalues and normalized eigenfunctions of the operator

$$
\begin{gathered}
\hat{H}(\mathbf{r})=\left(-i \nabla-\frac{2 e}{\hbar c} \mathbf{A}(\mathbf{r})\right)^{2}, \\
\hat{H}(\mathbf{r}) \psi_{j}(\mathbf{r})=\varepsilon_{j} \psi_{j}(\mathbf{r}),
\end{gathered}
$$

$$
\int_{-\infty}^{+\infty} \int_{-\infty}^{+\infty} \psi_{j}(\mathbf{r}) \psi_{j^{\prime}}^{*}(\mathbf{r}) d^{2} \mathbf{r}=\delta_{j, j^{\prime}}
$$

Substituting the expression (27) into the Eq. (10) we find the quantum correction to the conductance

$$
\Delta g(\mathbf{r})=-\frac{2 e^{2}}{\pi \hbar} \sum_{j} \frac{\left|\psi_{j}(\mathbf{r})\right|^{2}}{\varepsilon_{j}+\frac{1}{D \tau_{\varphi}}} e^{-D \tau\left(\varepsilon_{j}+\frac{1}{D \tau_{\varphi}}\right)} .
$$

Thus, the correction to the conductance is defined only by the spectrum and by the set of eigenfunctions of the operator $\hat{H}(\mathbf{r})$. Further we will be interested only in spatially averaged quantum correction to the conductance which defines the voltage between the sample contacts. Then taking into account the normalization condition for eigenfunctions we obtain:

$$
\langle\Delta g\rangle=-\frac{2 e^{2}}{\pi \hbar} \sum_{j} \frac{e^{-D \tau\left(\varepsilon_{j}+\frac{1}{D \tau_{\varphi}}\right)}}{\varepsilon_{j}+\frac{1}{D \tau_{\varphi}}} .
$$

To calculate the spectrum of the operator $\hat{H}(\mathbf{r})$ let us expand the magnetic field into the Fourier series

$$
B_{z}(\mathbf{r})=H+\sum_{\mathbf{b}_{n} \neq 0} H_{n} e^{i \mathbf{b}_{n} \mathbf{r}} .
$$

We start our analysis from the case of magnetic field with zero spatial average, i.e. we put $H=0$. The corresponding vector potential can also be written in the form of Fourier series

$$
\mathbf{A}(\mathbf{r})=\sum_{\mathbf{b}_{n} \neq 0} \mathbf{A}_{n} e^{i \mathbf{b}_{n} \mathbf{r}}
$$

Choosing the vector potential in the Lorentz gauge $\operatorname{div} \mathbf{A}=0$, so that

$$
\mathbf{A}_{n}=i \frac{\left[\mathbf{b}_{n}, \mathbf{H}_{n}\right]}{\mathbf{b}_{n}^{2}},
$$

we obtain the following expression for the operator $\hat{H}(\mathbf{r})$ :

$$
\begin{gathered}
\hat{H}(\mathbf{r})=-\nabla^{2}-\frac{4 e}{\hbar c} \sum_{\mathbf{b}_{n} \neq 0} H_{n} e^{i \mathbf{b}_{n} \mathbf{r}} \alpha_{n} \nabla \\
+\frac{4 e^{2}}{\hbar^{2} c^{2}} \sum_{\mathbf{b}_{n} \neq 0} Q_{n} e^{i \mathbf{b}_{n} \mathbf{r}}+\frac{4 e^{2}}{\hbar^{2} c^{2}} \sum_{\mathbf{b}_{n} \neq 0} \frac{\left|H_{n}\right|^{2}}{\mathbf{b}_{n}^{2}},
\end{gathered}
$$

where

$$
\alpha_{n}=\frac{\left[\mathbf{b}_{n}, \mathbf{z}_{0}\right]}{\mathbf{b}_{n}^{2}}
$$




$$
Q_{n}=\sum_{m \neq 0, m \neq n} \frac{H_{m} H_{n-m}}{\left(\mathbf{b}_{n}-\mathbf{b}_{m}\right)^{2}}\left(1-\frac{\left(\mathbf{b}_{n}, \mathbf{b}_{m}\right)}{\mathbf{b}_{m}^{2}}\right) .
$$

The Hamiltonian is translationally invariant $(\hat{H}(\mathbf{r}+$ $\left.\left.\mathbf{R}_{n}\right)=\hat{H}(\mathbf{r})\right)$ and its eigenfunctions satisfy the Bloch theorem:

$$
\psi_{\mathbf{k}}(\mathbf{r})=\sum_{\mathbf{b}_{n}} u_{n} e^{i\left(\mathbf{k}+\mathbf{b}_{n}\right) \mathbf{r}} .
$$

Substituting the expression (38) into Eq. (29) and introducing the value

$$
\varepsilon^{\prime}=\varepsilon-\frac{4 e^{2}}{\hbar^{2} c^{2}} \sum_{\mathbf{b}_{n} \neq 0} \frac{\left|H_{n}\right|^{2}}{\mathbf{b}_{n}^{2}},
$$

we find the following equation for the amplitudes $u_{n}$ :

$$
\begin{gathered}
{\left[\left(\mathbf{k}+\mathbf{b}_{n}\right)^{2}-\varepsilon^{\prime}\right] u_{n}+\frac{4 e^{2}}{\hbar^{2} c^{2}} \sum_{\mathbf{b}_{m} \neq 0} Q_{m} u_{n-m}} \\
-\frac{4 i e}{\hbar c} \sum_{\mathbf{b}_{m} \neq 0} u_{n-m} H_{m} \alpha_{m}\left(\mathbf{k}+\mathbf{b}_{n}-\mathbf{b}_{m}\right)=0 .
\end{gathered}
$$

In the absence of the magnetic field only the amplitude $u_{n}$ corresponding to $n=0$ is nonzero $\left(u_{n=0} \neq 0\right.$ and $\left.u_{n \neq 0}=0\right)$. Therefore, in this case the spectrum has the form $\varepsilon(\mathbf{k})=\mathbf{k}^{2}$.

Let us consider the dephasing time at low temperatures when $L_{\varphi} \gg d$ ( $d$ is the characteristic scale of magnetic field inhomogeneity). We also restrict ourselves to the case of low amplitude of the magnetic field so that $L_{H_{0}} \gg d\left(L_{H_{0}}=\sqrt{\Phi_{0} / H_{0}}\right)$. Then, as it is seen from the Eq. (31), the zero temperature divergence of the conductance correction comes from the region of low $\varepsilon$ which corresponds to the region of low $|\mathbf{k}|$. Taking into account the condition $d \ll L_{H_{0}}$ we will assume that the region $|\mathbf{k}| \ll\left|\mathbf{b}_{n}\right|$ gives the main contribution to the low temperature correction. In this case the spectrum of the operator $\hat{H}(\mathbf{r})$ can be calculated in the "nearly free electron" approximation.

In the presence of magnetic field the spectrum can be written in the form $\varepsilon^{\prime}(\mathbf{k})=\mathbf{k}^{2}+\varepsilon^{(1)}(\mathbf{k})+\varepsilon^{(2)}(\mathbf{k})$, where $\varepsilon^{(1)}$ is proportional to the field amplitude and $\varepsilon^{(2)}$ is proportional to the square of the field's amplitude.

The first order correction to the zero field spectrum is equal to the second term in the expression (39) but with the opposite sign. Thus, the correction $\varepsilon^{(1)}$ in the spectrum $\varepsilon^{\prime}$ is zero. The second-order correction $\varepsilon^{(2)}$ has the following form:

$$
\varepsilon^{(2)}=-\frac{16 e^{2}}{\hbar^{2} c^{2}} \sum_{\mathbf{b}_{n} \neq 0} \frac{\left|H_{n}\right|^{2}\left(\alpha_{n}, \mathbf{k}\right)^{2}}{\mathbf{b}_{n}^{2}} .
$$

Thus, the spectrum reads

$$
\begin{aligned}
\varepsilon(\mathbf{k})=\mathbf{k}^{2} & -\frac{16 e^{2}}{\hbar^{2} c^{2}} \sum_{\mathbf{b}_{n} \neq 0} \frac{\left|H_{n}\right|^{2}\left(\mathbf{k},\left[\mathbf{b}_{n}, \mathbf{z}_{0}\right]\right)^{2}}{\mathbf{b}_{n}^{6}} \\
& +\frac{4 e^{2}}{\hbar^{2} c^{2}} \sum_{\mathbf{b}_{n} \neq 0} \frac{\left|H_{n}\right|^{2}}{\mathbf{b}_{n}^{2}} .
\end{aligned}
$$

The second term in the Eq. (42) is of the order of $k^{2}\left(d / L_{H_{0}}\right)^{4} \ll k^{2}$ and leads to renormalization of the "effective mass" in a quadratic spectrum $\varepsilon(\mathbf{k}) \propto k^{2}$. This change in the "effective mass" does not affect the zero temperature divergence of the weak-localization correction to the conductance and further will be neglected. Thus, the resulting spectrum has the form

$$
\varepsilon(\mathbf{k})=\mathbf{k}^{2}+\frac{4 e^{2}}{\hbar^{2} c^{2}} \sum_{\mathbf{b}_{n} \neq 0} \frac{\left|H_{n}\right|^{2}}{\mathbf{b}_{n}^{2}} .
$$

Note that the second term in Eq. (43) makes an important contribution to the dephasing time. The effective dephasing time has the form

$$
\frac{1}{\tau_{B}}=\frac{1}{\tau_{\varphi}}+\frac{4 e^{2} D}{\hbar^{2} c^{2}} \sum_{\mathbf{b}_{n} \neq 0} \frac{\left|H_{n}\right|^{2}}{\mathbf{b}_{n}^{2}} .
$$

From the expression (44) one can see that for arbitrarily small magnetic field the low temperature divergence of quantum correction to the conductance is cut off by the finite effective dephasing time $\tau_{B}$. The corresponding expression for quantum correction to the conductance reads:

$$
\langle\Delta g\rangle \approx \frac{e^{2}}{2 \pi^{2} \hbar} \ln \left[\frac{\tau}{\tau_{\varphi}}+\frac{4 e^{2} D \tau}{\hbar^{2} c^{2}} \sum_{\mathbf{b}_{n} \neq 0} \frac{\left|H_{n}\right|^{2}}{\mathbf{b}_{n}^{2}}\right] .
$$

The logarithmic term dominates in the weak-localization correction which allows us to consider the contribution of magnetic field only in the argument of logarithmic function, and we neglect small additional corrections to the expression (45), which are also caused by the magnetic field.

In the limit of zero temperature the value $\tau_{B}^{-1}$ is proportional to the square of amplitude of magnetic field in a sharp contrast to the case of homogeneous field where $t_{H}^{-1} \sim H$. This analytical result is in agreement with the qualitative estimate obtained in Ref. 9.

Now we proceed with the analysis of the case of periodic magnetic field with nonzero but small spatial average (i.e. $H \neq 0$ and $H S_{0} \ll \Phi_{0}, S_{0}$ is the area of the unit cell defined by the basic vectors $\mathbf{a}_{1}$ and $\mathbf{a}_{2}$ ) at low temperatures when $L_{\varphi} \gg d$. The vector potential is given by

$$
\mathbf{A}(\mathbf{r})=\frac{1}{2}[\mathbf{H}, \mathbf{r}]+\sum_{\mathbf{b}_{n} \neq 0} \mathbf{A}_{n} e^{i \mathbf{b}_{n} \mathbf{r}}
$$


where $\mathbf{H}=H \mathbf{z}_{0}$. To obtain the spectrum of the operator $\hat{H}(\mathbf{r})$ we can first admit that the vector potential $\mathbf{A}_{0}(\mathbf{r})=$ $\frac{1}{2}[\mathbf{H}, \mathbf{r}]$ corresponding to the homogeneous component $H$ of magnetic field is constant on the characteristic scales of a periodic magnetic field $\stackrel{23}{\underline{n}}$ The spectrum of the operator $\hat{H}(\mathbf{r})$ has the following form (see (43)):

$$
\varepsilon(\mathbf{k})=\left(\mathbf{k}-\frac{2 e}{\hbar c} \mathbf{A}_{0}\right)^{2}+\frac{4 e^{2}}{\hbar^{2} c^{2}} \sum_{\mathbf{b}_{n} \neq 0} \frac{\left|H_{n}\right|^{2}}{\mathbf{b}_{n}^{2}} .
$$

Proceeding with the analysis in the momentum space one needs to restore the commutation relations for the components of quasi-momentum $\mathbf{k}$ and the components of the radius vector operator $\hat{\mathbf{r}}=i \partial / \partial \mathbf{k}$. Then the spectrum (47) transforms into a new effective operator, which can be reduced to the harmonic oscillator hamiltonian. The spectrum of this effective hamiltonian has the form

$$
\varepsilon_{m}=\frac{2 e H}{\hbar c}(2 m+1)+\frac{4 e^{2}}{\hbar^{2} c^{2}} \sum_{\mathbf{b}_{n} \neq 0} \frac{\left|H_{n}\right|^{2}}{\mathbf{b}_{n}^{2}},
$$

where $m$ is a nonzero integer number. Finally carrying out the summation over $m$ in the Eq. (31) we obtain

$$
\begin{aligned}
&\langle\Delta g\rangle=-\frac{e^{2}}{2 \pi^{2} \hbar} \\
& \times\left\{\psi\left(\frac{1}{2}+\frac{\hbar c}{4 e H D \tau}\right)-\psi\left(\frac{1}{2}+\frac{\hbar c}{4 e H D \tau_{B}}\right)\right\},
\end{aligned}
$$

where the value $\tau_{B}$ is defined by the expression (44). Expanding the expression (49) in the limit $H D \tau_{B} / \Phi_{0} \ll 1$, we obtain

$$
\langle\Delta g\rangle \approx-\frac{e^{2}}{2 \pi^{2} \hbar} \ln \left(\frac{\tau_{B}}{\tau}\right)+\frac{e^{2}}{3 \pi^{2} \hbar}\left(\frac{e H D \tau_{B}}{\hbar c}\right)^{2} .
$$

The expressions (49) and (50) formally coincide with the ones for the homogeneous field, but in a modulated magnetic field the dephasing time $\tau_{B}$ is determined by the amplitude of modulation.

\section{STRONG MAGNETIC FIELDS. POSITIVE MAGNETORESISTANCE}

We now proceed with the consideration of the strong field limit and focus on the possibility to change the sign of magnetoresistance of $2 \mathrm{DEG}$ in the presence of a modulated magnetic field. Specifically, we consider a ferromagnetic film/2DEG system placed in the external magnetic field perpendicular to the $2 \mathrm{DEG}$ plane. Let the ferromagnetic film have a periodic stripe domain structure. We will assume that the film of 2DEG is thin enough to consider only the $z$ component $H_{z}$ of magnetic field depending only on $x$ coordinate along the sample surface.
We will denote the external field as $H$ and the absolute value of the periodic field of stripe structure by $H_{0}$. Further the description of the weak-localization correction to the conductance will be developed on the basis of local approximation. This approximation is correct when the electron dephasing length is less than the characteristic scale of inhomogeneous magnetic field. These conditions mean that $d \gg \min \left\{L_{\varphi}, L_{B}\right\}$ (the gray region in Fig. (1). Note that the effect of positive magnetoresistance can be observed only in the region of parameters where the local approximation is applicable. Indeed, the Eqs. (21) and (50) show that in the opposite limit the second derivative $\langle\Delta g(H)\rangle$ at $H=0$ is positive and, as a result, the magnetoresistance is negative.

The effect of positive magnetoresistance strongly depends on the magnetic field configuration. We assume for simplicity the thickness of ferromagnetic layer to exceed strongly the period of stripe structure $d$. In this case for hybrid structures F/2DEG the spatial configuration of magnetic field in the region of 2DEG depends mostly on the thickness of the spacer between 2DEG and ferromagnetic film. If the spacer is much thinner than the period of stripe structure $d$ then the distribution of the $z$-component $B_{z}(\mathbf{r})$ of the magnetic field in 2DEG approximately has the form of meander. In the opposite case, when the spacer thickness is much larger than the spatial period of the domain structure, the magnetic field profile is smeared. On a qualitative level one can describe this limit considering a sinusoidal field profile.

\section{A. Periodic magnetic field in the form of meander}

The periodic magnetic field in the form of meander is the simplest configuration of magnetic field which reveals the effect of positive magnetoresistance. The external homogeneous magnetic field applied to the system leads to the suppression of weak localization in the regions where the sign of the external field coincides with the one of the periodic field component. In opposite, the external field results in the increase of the interference corrections in the domains where the sign of these field components are different. The competition between these two effects defines the resulting dependence of the averaged conductance vs an external homogeneous field. If the increase in the weak-localization correction dominates then the resulting dependence of averaged conductance vs external homogeneous magnetic field is decreasing. In this case one can conclude that 2DEG has positive magnetoresistance.

Let us search for the region of parameters, where the effect of positive magnetoresistance can be observed. Within the local approximation the averaged conductance is defined by the following expression:

$$
\left\langle g_{m}(h)\right\rangle=\frac{1}{2}\left[g\left(h+h_{0}\right)+g\left(h-h_{0}\right)\right],
$$



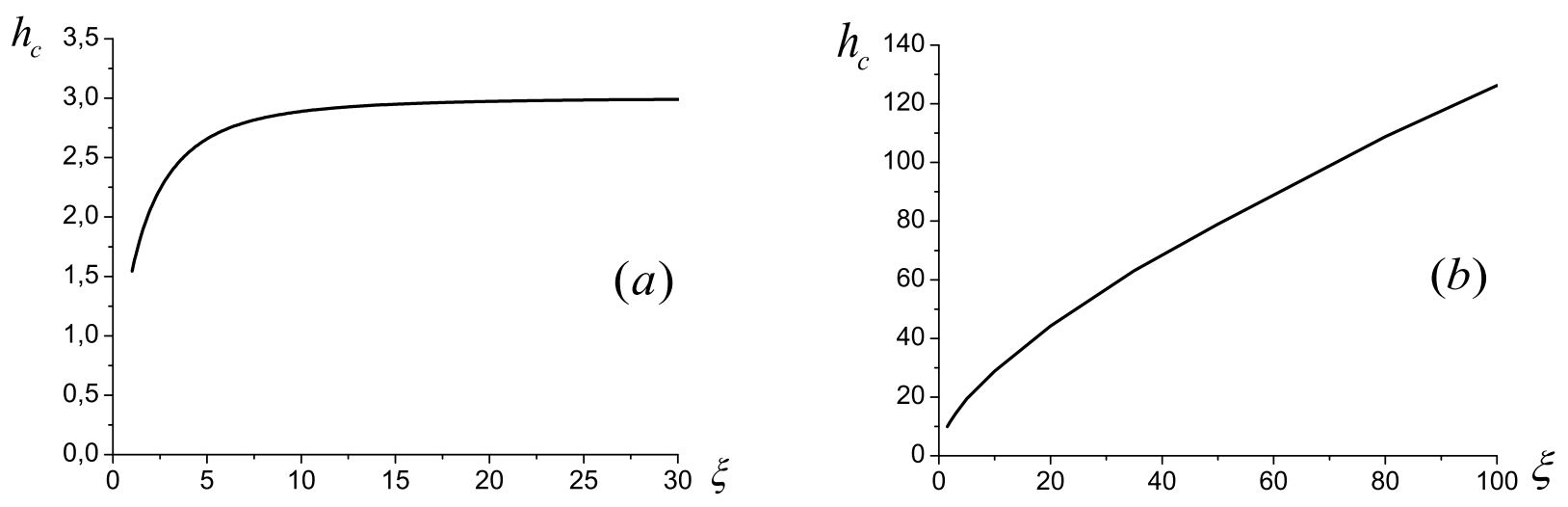

FIG. 4: The dependencies of the critical magnetic field amplitude $h_{c}$, which separates the regions of positive and negative magnetoresistance, vs the parameter $\xi=\tau_{\varphi} / \tau$ : (a) meander field profile, (b) sinusoidal field profile.

where $h_{0}=\frac{4 e H_{0} D \tau_{\varphi}}{\hbar c}$

$$
\begin{gathered}
g(H)=g_{D}-\frac{e^{2}}{2 \pi^{2} \hbar} \times \\
\left\{\psi\left(\frac{1}{2}+\frac{\hbar c}{4 e|H| D \tau}\right)-\psi\left(\frac{1}{2}+\frac{\hbar c}{4 e|H| D \tau_{\varphi}}\right)\right\}
\end{gathered}
$$

and $g_{D}$ is the Drude conductance. Introducing dimensionless variables we obtain:

$$
g(h)=g_{D}-g_{0}\left\{\psi\left(\frac{1}{2}+\frac{\xi}{|h|}\right)-\psi\left(\frac{1}{2}+\frac{1}{|h|}\right)\right\},
$$

where $g_{0}=\frac{e^{2}}{2 \pi^{2} \hbar}, \xi=\frac{\tau_{\varphi}}{\tau}, h=\frac{4 e H D \tau_{\varphi}}{\hbar c}$. Expanding the expression (51) into the Taylor's series for $h \ll h_{0}$ we find

$$
\left\langle g_{m}(h)\right\rangle \approx g\left(h_{0}\right)+\frac{1}{2} g^{\prime \prime}\left(h_{0}\right) h^{2} .
$$

One can see that the positive magnetoresistance is realized for $h_{0}$, which satisfies the condition

$$
g^{\prime \prime}\left(h_{0}\right)<0 .
$$

This condition is realized when the amplitude of periodic magnetic field is larger than some critical value $h_{c}$, which depends on the parameter $\xi$. The dependence $h_{c}(\xi)$ for the meander configuration of periodic magnetic field is shown in Fig. [4(a). For $\xi \gg 1$ the boundary of the positive magnetoresistance region is defined by the condition $h_{c} \approx 3$.

These conclusions are based on the equation (3) and, thus, are valid only in the diffusive limit. The domain of applicability of the diffusive approximation is defined by the condition $L_{B} \gg l$, where $l$ is an elastic scattering length. In the limit $\lambda_{F} \ll L_{B} \ll l$ (where $\lambda_{F}$ is the Fermi wave-length) the weak-localization is fully suppressed and the conductance approaches the Drude value.

The dependencies of the averaged conductance of 2DEG vs the external homogeneous field at different amplitudes of periodic field are shown in Fig. 5(a) for $\xi=100$. One can see that for $h_{0} \gg h_{c}(\xi)$ these dependencies have the sharp dips with minima at $h=h_{0}$.

\section{B. Periodic magnetic field in the form of cosine}

As a second example we consider the effect of positive magnetoresistance in the sinusoidal profile of the $z$-component of the magnetic field

$$
H_{z}(x)=H_{0} \cos (\pi x / d) .
$$

The expression for the averaged conductance does not depend on the period of magnetic field $d$ and has the following form:

$$
\begin{gathered}
\left\langle g_{c}(h)\right\rangle=g_{D}-g_{0} \\
\times \int_{0}^{1}\left\{\psi\left(\frac{1}{2}+\frac{\xi}{\left|h+h_{0} \cos (\pi \rho)\right|}\right)-\psi\left(\frac{1}{2}+\frac{1}{\left|h+h_{0} \cos (\pi \rho)\right|}\right)\right\} d \rho .
\end{gathered}
$$

Here $h_{0}=\frac{4 e H_{0} D \tau_{\varphi}}{\hbar c}$. The set of dependencies of $\left\langle g_{c}(h)\right\rangle$ for different magnitudes of amplitude $h_{0}$ is shown in Fig. 5(b). From the comparison between Fig. 5(a) and Fig. (5) one can see that in periodic magnetic field with sinusoidal profile the effect of positive magnetoresistance is weaker than in the case of meander profile. This is caused by the fact that for the sinusoidal profile the regions where the external and periodic fields have opposite directions shrink with the external field increasing. 

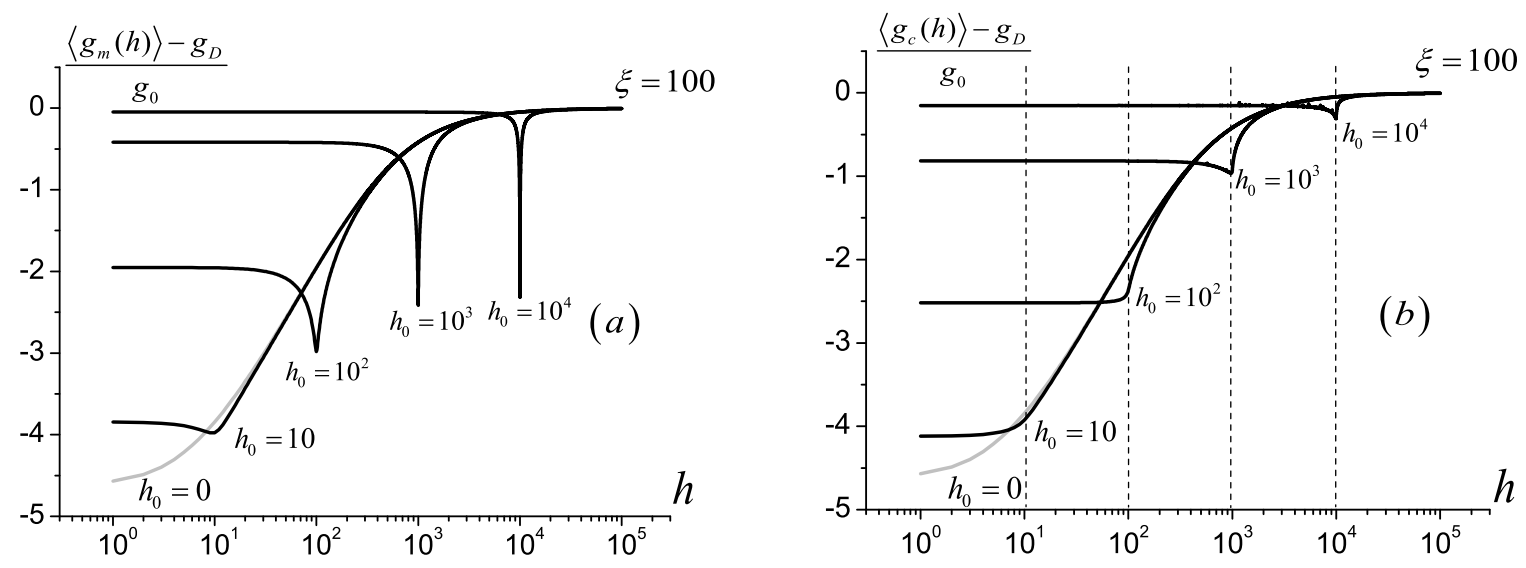

FIG. 5: The averaged conductance of 2DEG vs the external homogeneous field at different amplitudes of the periodic field $h_{0}$ and at $\xi=100$ : (a) meander field profile, (b) sinusoidal field profile.

In Fig. 5(b) the amplitude of the periodic magnetic field is shown by the vertical dotted line. One can see that for $h \approx h_{0}$ the behavior of $\left\langle g_{c}(h)\right\rangle$ changes qualitatively. Even for high amplitude of the periodic magnetic field $h_{0}$ in the region $h<h_{0}$ the conductance deviates from the Drude value. This is caused by the incomplete destruction of the interference near the field zero points even at rather high $h_{0}$ values.

The expression (54) allows to find the condition of positive magnetoresistance in combined cosinusoidal and homogeneous magnetic fields. The magnetoresistance peak appears provided

$$
\left.\frac{\partial^{2} g_{c}(h)}{\partial h^{2}}\right|_{h=0}<0
$$

which gives us the condition

$$
\int_{0}^{1} g^{\prime \prime}\left(h_{0} \beta\right) \frac{d \beta}{\sqrt{1-\beta^{2}}}<0 .
$$

Here the function $g$ is defined by the expression (52). The condition (55) is satisfied when the periodic magnetic field amplitude $h_{0}$ is larger than the critical value $h_{c}(\xi)$. The dependence $h_{c}(\xi)$ for sinusoidal profile of periodic magnetic field is shown in Fig. 4(b). One can observe a clear difference between two model profiles: contrary to the meander case the critical field diverges at large $\xi$ values.

\section{SUMMARY}

To sum up, we have investigated the influence of inhomogeneous magnetic fields on the weak localization phenomenon in 2DEG systems. In the low field limit we have carried out a perturbative analysis of the conductance behavior at high temperatures and developed an analytical procedure to find a renormalization of the dephasing rate at low temperatures. In the high field limit we have justified the validity of the local approximation and have used this approach to calculate the averaged conductance for particular model field profiles. It is found that the systems with modulated magnetic field profiles provide a possibility to observe the effect of positive magnetoresistance. We have showed that the positive magnetoresistance in ferromagnetic film/2DEG systems can be observed experimentally provided the amplitude of the field modulation exceeds a certain critical value depending on the system parameters.

Finally, we consider some estimates for existing experimental systems. We take here the 2DEG system in the GaAs/AlGaAs heterostructure with high electron mobility as a typical example (see Ref. 25). This system is characterized by the following typical parameters: $\hbar c(4 e D \tau)^{-1}=0.7 O e$ and $\hbar c\left(4 e D \tau_{\varphi}\right)^{-1}=0.2 O e$. The typical amplitude of the magnetic field induced by the ferromagnetic film with a domain structure is of the order of $H_{0} \sim 10^{2}-10^{3} \mathrm{Oe}$ (see Ref. 2). For the meander distribution of the magnetic field with such amplitude the height of the magnetoresistance peak can reach one half of the weak-localization correction in zero field.

\section{ACKNOWLEDGEMENTS}

This work is supported by the RFBR, RAS under the Federal Scientific Program "Quantum physics of condensed matter", the "Dynasty" Foundation, the Russian Agency of Education under the Federal Program "Scientific and educational personnel of innovative Russia in 2009-2013". 
1 A. I. Buzdin, Rev. Mod. Phys. 77, 935 (2005).

2 A. Yu. Aladyshkin, A. V. Silhanek, W. Gillijns and V. V. Moshchalkov, Supercond. Sci. Technol. 22, 053001 (2009).

3 S. Shakravarty and A. Schmid, Physics Reports 140, No.4, 1986.

4 J. Rammer, and A. L. Shelankov, Phys. Rev. B 36, 3135 (1987).

5 S. J. Bending, K. von Klitzing, and K. Ploog, Phys. Lett. 65, 1060 (1990).

6 S. J. Bending, K. von Klitzing, and K. Ploog, Phys. Rev. B 42, 9859 (1990).

7 S. J. Bending, A. K. Geim, Phys. Rev. B 46, 14912 (1992).

8 S. J. Bending, Phys. Rev. B 50, 17621 (1994).

9 Xiao-Bing Wang, Phys. Rev. B 65, 115303 (2002).

10 G. M. Gusev, U. Gennser, X. Kleber, D. K. Maude, J. C. Portal, D. I. Lubyshev, P. Basmaji, M. de P. A. Silva, J. C. Rossi, Yu. V. Nastaushev, Phys. Rev. B 53, 13641 (1996).

11 H. Mathur and H. U. Baranger, Phys. Rev. B 64, 235325 (2001).

12 P. D. Ye, D. Weiss, R. R. Gerhardts, M. Seeger, K. von Klitzing, K. Eberl, and H. Nickel, Phys. Rev. Lett. 74, 3013 (1995).

13 Deng Ping Xue and Gang Xiao, Phys. Rev. B 45, 5986 (1992).
14 I. S. Ibrahim and F. M. Peeters, Phys. Rev. B 52, 17321 (1995).

15 A. Shelankov, Phys. Rev. B 62, 3196 (2000).

16 F. B. Mancoff, R. M. Clarke, C. M. Marcus, S. C. Zhang, K. Campman, A. C. Gossard, Phys Rev. B 51, 13269 (1995).

17 S. Hikami, A. I. Larkin and Y. Nagaoka, Prog. Theor. Phys. 63, 707 (1980).

18 G. Bergmann, Phys. Rev. Lett. 48, 1946 (1982).

19 Per Hedegard, and Anders Smith, Phys. Rev. B 51, 10869 (1995).

20 F. M. Peeters and P. Vasilopoulos, Phys. Rev. B 47, 1466 (1993).

21 A. Matulis and F. M. Peeters, Phys. Rev. B 62, 91 (2000).

22 A. K. Geim, S. J. Bending, I. V. Grigorieva, M. G. Blamire, Phys. Rev. B 49, 5749 (1994).

23 R. Peierls, Z. Phys. 80, 763 (1933).

24 B. L. Altshuler and A. G. Aronov, Electron-Electron Interactions in Disordered Systems, edited by A. L. Efros and M. Pollak (North-Holland, Amsterdam, 1985).

25 R. Taboryski and P. E. Lindelof, Semicond. Sci. Technol. 5, 933-946 (1990). 